\title{
Upregulation of CD54 and downregulation of HLA-ABC contribute to the novel enhancement of the susceptibility of HL-60 cells to NK cell-mediated cytolysis induced by ATRA plus VPA
}

\author{
HUIJUAN ZOU ${ }^{1 *}$, LIANLIAN LI $^{1 *}$, YANG HAN ${ }^{1,2^{*}}$, RUIPING MA $^{3}$, QIONG LIAO $^{1,2}$, JING TIAN $^{1,2}$, \\ XIAOYU ZHANG ${ }^{1}$, XIA REN $^{1}$, GUANHUA SONG ${ }^{1}$, QIANG GUO $^{1}$, XIA LI $^{1}$, \\ HUIFANG DING ${ }^{4}$ and GUOSHENG JIANG ${ }^{1}$
}

\begin{abstract}
${ }^{1}$ Department of Hemato-Oncology, Institute of Basic Medicine, Shandong Academy of Medical Sciences, Key Medical Laboratory for Tumor Immunology and Traditional Chinese Medicine Immunology of Shandong, Jinan, Shandong 250062; ${ }^{2}$ School of Medicine and Life Sciences, University of Jinan-Shandong Academy of Medical Sciences, Jinan, Shandong 250062; ${ }^{3}$ Department of Oncology, Shandong Provincial Qianfoshan Hospital, Jinan, Shandong 250014;

${ }^{4}$ Department of Hematology, Shengli Oilfield Central Hospital, Dongying, Shandong 257034, P.R. China
\end{abstract}

Received May 6, 2016; Accepted June 23, 2016

DOI: $10.3892 /$ or.2016.5212

\begin{abstract}
Enhancement of the susceptibility of HL-60 cells to NK cell-mediated cytolysis induced by all-trans-retinoic acid (ATRA) plus valproate (VPA) was evaluated. In addition to the synergistic effect of ATRA plus VPA on HL-60 cells, the optimal concentration of $1 \mathrm{mM}$ VPA plus $0.5 \mu \mathrm{M}$ ATRA increased the cytotoxic sensitivity of HL-60 cells to NK cells. The expression of the activated receptors NKp30 and NKG2D on NK-92 cells was higher compared with the levels noted for the other receptors, and the expression of NKG2D ligands MICA/B on HL-60 cells was not significantly upregulated in the ATRA plus VPA goup compared with the control. Moreover, it was observed that the ligands of NKp30 on HL-60 cells presented the same variation trend. As to the co-stimulatory and adhesion molecules on NK-92 and their ligands on HL-60 cells post exposure to ATRA and VPA alone or their combination, there was no obvious change in the expression of CD112, CD48 and CD70 on the HL-60 cells.
\end{abstract}

Correspondence to: Dr Huifang Ding, Department of Hematology, Shengli Oilfield Central Hospital, 31 Jinan Road, Dongying, Shandong 257034, P.R. China

E-mail: dingmx2008@126.com

Dr Guosheng Jiang, Department of Hemato-Oncology, Institute of Basic Medicine, Shandong Academy of Medical Sciences, 18877 Jingshi Road, Jinan, Shandong 250062, P.R. China

E-mail: jianggsh@hotmail.com

*Contributed equally

Key words: ATRA, VPA, HL-60 cells, differentiation, apoptosis, NK cells
However, the expression of CD54 on HL-60 cells was significantly upregulated. In contrast, the expression of NKG2A ligands HLA-ABC on HL-60 cells was obviously downregulated. In addition, the expression of HLA-E on the HL-60 cells in the group treated with ATRA plus VPA was not significantly increased. In conclusion, the combination of VPA and ATRA not only induced the differentiation of HL-60 cells, but also induced enhancement of the sensitivity of HL-60 cells to NK cells by downregulating the expression of HLA-ABC and upregulating the expression of CD54, but not MICA/MICB. The results provide experimental and theoretical basis for the clinical combination of a low-dose of ATRA plus VPA for the treatment of leukemia.

\section{Introduction}

Acute myeloid leukemia (AML), an aggressive hematological malignancy which seriously endangers human health, is characterized by a blockage of terminal differentiation of primitive hematopoietic stem and progenitor cells at early stages in myelopoiesis, leading to increased proliferation of immature myeloid progenitors (1). In 1986, all-trans-retinoic acid (ATRA) was approved to treat patients with acute promyelocytic leukemia (APL) and most patients with APL achieved complete remission (2). Successively, $\mathrm{As}_{2} \mathrm{O}_{3}$ was also found to induce differentiation and apoptosis of leukemia cells and caused remission in most APL patients $(3,4)$. Unfortunately, treatments using ATRA or $\mathrm{As}_{2} \mathrm{O}_{3}$ have the disadvantages of a high recurrence rate and drug resistance $(5,6)$. Therefore, it is urgent to explore novel agents or their combination to enhance the induction of differentiation or reduce the relapse rate of APL patients.

Histone deacetylase inhibitors (HDACIs) have been suggested for use as a new type of differentiation-inducing therapy for leukemia (7), and an increasing number of HDACIs 
Table I. Primary antibodies used in the present study.

\begin{tabular}{|c|c|c|c|c|}
\hline Name & Fluorescent & Antibody isotype & Catalog no. & Company \\
\hline CD11b (Mac-1) & Alexa Flour 488 & Mouse IgG1, $\kappa$ & 557701 & BD \\
\hline $\mathrm{MICA} / \mathrm{B}$ & PE & Mouse IgG2a, $\kappa$ & 558352 & $\mathrm{BD}$ \\
\hline HLA-ABC & $\mathrm{PE}$ & Mouse $\operatorname{IgG} 1, \kappa$ & 555553 & $\mathrm{BD}$ \\
\hline CD11a (LFA-1) & PE & Mouse $\operatorname{IgG} 1, \kappa$ & 555384 & $\mathrm{BD}$ \\
\hline CD48 & FITC & Mouse IgG1, $\kappa$ & 555759 & $\mathrm{BD}$ \\
\hline CD54 (ICAM-1) & $\mathrm{PE}$ & Mouse $\operatorname{IgG} 1, \kappa$ & 555511 & $\mathrm{BD}$ \\
\hline CD70 & $\mathrm{PE}$ & Mouse IgG3, $\kappa$ & 555835 & $\mathrm{BD}$ \\
\hline $\mathrm{CD} 85 \mathrm{j}$ & FITC & Mouse IgG2b, $\kappa$ & 555942 & $\mathrm{BD}$ \\
\hline CD112 & PE & Mouse IgG1, $\kappa$ & 551057 & BD \\
\hline CD244 (2B4) & PE & Mouse IgG2a, $\kappa$ & 550816 & $\mathrm{BD}$ \\
\hline CD226 & $\mathrm{PE}$ & Mouse $\operatorname{IgG} 1, \kappa$ & 559789 & $\mathrm{BD}$ \\
\hline NKG2A (CD159a) & PE & Mouse IgG2a & FAB105P & $R \& D$ \\
\hline NKG2C (CD159c) & $\mathrm{PE}$ & Mouse IgG1 & FAB138P & $R \& D$ \\
\hline NKG2D (CD314) & PE & Mouse IgG1, $\kappa$ & 557940 & $\mathrm{BD}$ \\
\hline NKp30 (CD337) & PE & Mouse IgG1, $\kappa$ & 558407 & $\mathrm{BD}$ \\
\hline NKp44 & PE & Mouse IgG1, $\kappa$ & 558563 & $\mathrm{BD}$ \\
\hline NKp46 & PE & Mouse IgG1, $\kappa$ & 557991 & $\mathrm{BD}$ \\
\hline HLA-E & $\mathrm{PE}$ & Mouse IgG1 & ab11821 & Abcam \\
\hline
\end{tabular}

BD, BD Biosciences (San Diego, CA, USA); R\&D, R\&D Systems, Inc. (Minneapolis, MN, USA); Abcam, Cambridge, MA, USA.

used in the clinic including short-chain fatty acids (butyric acid) and hydroxamic acids (TSA and SAHA) were investigated for the treatment of tumors and leukemias. Valproate (VPA) as one of the HDACIs alone or combined with ATRA was used in the treatment of patients with leukemia and achieved good clinical efficacy (8-17). Yet, it was not clear whether the combination of VPA plus ATRA interfers with the function of immune cells.

Natural killer (NK) cells, the primary effector cells of the innate immune system, play an important role in antitumor and anti-viral infection. NKG2D which crosslinks with its ligands (NKG2DL) is the unique activating receptor of NK cells, which triggers the cytotoxic activity of NK cells. Armeanu et al found that the mRNA and protein of NKG2D ligands in liver cancer cells were increased after exposure to VPA and it may significantly enhance the NK sensitivity of NK-92 cells to HepG2 cells (18), and the enhanced killing effect of NK cells was mediated by upregulation of NKG2D ligands on the tumor cells. Zhang et al also suggested that sodium butyrate (SB) and VPA upregulated the mRNA and protein expression of MHC I molecule-associated proteins (MICA and MICB) in HeLa and HepG2 cells, resulting in the enhancement of sensitivity of tumor cells to NK cells (19). Of the various myeloid cell lines examined, a chronic myelogenous leukemia cell line OUN-1 showed the most prominent upregulation of MICA/B and ULBP2 in response to VPA or hydroxyurea (HU). OUN-1 cells treated with VPA plus HU were more susceptible to be killed by NK cells than untreated cells (20). In vivo administration of ATRA or the histone deacetylase inhibitor sodium VPA to patients with APL or M1 respectively, led to the induction of transcription and expression of NKG2DL at the surface of leukemic cells. Furthermore, upon therapy with ATRA or VPA, leukemic blasts acquire the ability to trigger lytic granule exocytosis by autologous $\mathrm{CD}^{+}$ $\mathrm{T}$ and NK lymphocytes. These findings indicate that ATRA and VPA may contribute to the activation of cytolytic effector lymphocytes in vivo (21). According to the above results, VPA has potential therapeutic effects by enhancing the immune response to leukemia. In the present study, the enhancement of susceptibility of HL-60 cells to NK cell-mediated cytolysis was firstly detected when the cooperation of ATRA and VPA in inducing differentiation was identified.

\section{Materials and methods}

Reagents. ATRA was dissolved with a small amount of dimethyl sulfoxide (DMSO) and a $10 \mathrm{mM}$ stock solution was prepared with RPMI-1640 medium (Gibco, Grand Island, NY, USA) and stored at $-20^{\circ} \mathrm{C}$. A $50 \mathrm{mg} / \mathrm{ml}$ stock solution of VPA (Sigma, St. Louis, MO, USA) was constructed with phosphatebuffered saline (PBS) and diluted with cell culture medium for use. Trypan blue, propidium iodide (PI) and carboxyfluorescein diacetate succinimidyl ester (CFSE) were purchased from Sigma. Annexin V fluorescein isothiocyanate (FITC) kit was obtained from BD Biosciences (San Diego, CA, USA). $\mathrm{Na}_{2}{ }^{51} \mathrm{CrO}_{4}$ for detection of $\mathrm{NK}$ cell activity was obtained from PerkinElmer. Antibodies for fluorescence-activating cell sorting (FACS) are listed in Table I.

Cell culture. The human acute APL HL-60 cell line was purchased from the Cell Bank of the Chinese Academy of Sciences (Shanghai, China) and was cultured with RPMI-1640 medium. Human natural killer NK-92 cells were conserved by the University of Science and Technology of China (Hefei, 
Anhui, China) and cultured in $\alpha$-MEM medium (Gibco). All of the cells were grown in medium supplemented with $10 \%$ fetal calf serum (HyClone, Logan, UT, USA), $100 \mathrm{IU} / \mathrm{ml}$ penicillin and $100 \mathrm{IU} / \mathrm{ml}$ streptomycin at $37^{\circ} \mathrm{C}$ in a $5 \% \mathrm{CO}_{2}$ humidified atmosphere. Logarithmically growing cells were exposed to drugs for the indicated time periods. Cells (at a density of $2 \times 10^{5}$ cell $/ \mathrm{ml}$ ) were treated with ATRA at concentrations of $0.1,0.5$ and $1.0 \mu \mathrm{M}$, and VPA at $0.5,1.0$ and $2.0 \mathrm{mM}$, alone or in combination. Cells treated with $0.1 \%$ (v/v) DMSO or RPMI1640 medium were used as control. The cells were incubated again at $37^{\circ} \mathrm{C}$ in $5 \% \mathrm{CO}_{2}$ and collected after 24,48 and $72 \mathrm{~h}$.

Effect of ATRA plus VPA on the proliferation of HL-60 cells. The effect of ATRA and VPA on the proliferation of HL-60 cells was assayed by trypan blue exclusion test. Briefly, the cells treated with the drugs as mentioned above were washed with cold 1X PBS buffer, and then re-suspended with PBS buffer. One part of the cell suspension was mixed with one part $0.4 \%$ trypan blue dye and incubated for 3-5 min. Cells were added to a cell counting plate after 3-5 min. Dye-cell mixture $(10 \mu \mathrm{l})$ was loaded to a clean cell counting chamber and stained cells which were the dead cells and unstained cells which were the live cells were counted under a microscope.

Cell cycle analysis. Following ATRA and/or VPA treatment, HL-60 cells were washed twice with ice-cold PBS, and fixed in cold $75 \%$ ethanol at $4^{\circ} \mathrm{C}$ for at least $24 \mathrm{~h}$. Then, the cells were re-suspended with PBS in $0.5 \mathrm{ml}$ of cell cycle buffer $(0.38 \mathrm{mM}$ Na-citrate, $0.5 \mathrm{mg} / \mathrm{ml}$ RNase A and $20 \mu \mathrm{g} / \mathrm{ml} \mathrm{PI}$ ) at $4^{\circ} \mathrm{C}$ for 30 min and analyzed using an EPICS XL flow cytometer with EXPO32 ${ }^{\mathrm{TM}}$ ADC software (Beckman Coulter, Miami, FL, USA).

FACS assays for the expression of markers on HL-60 and NK-92 cells. The drug-treated cells were collected and washed twice with ice-cold PBS, and then re-suspended with $100 \mu \mathrm{l}$ PBS. The cell suspensions were blocked with 5\% FBS for $30 \mathrm{~min}$ and incubated with fluorescent antibodies specific for surface markers including CD11b, CD11a, MICA/B, HLA-ABC, CD48, CD54, CD70, CD85j, CD112, CD244, CD226, NKG2A, NKG2C, NKG2D, NKp30, NKp44, NKp46 and HLA-E or isotype control antibodies for $30 \mathrm{~min}$ at $4^{\circ} \mathrm{C}$ in the dark. The cells were washed twice with cold PBS and re-suspended with $200 \mu \mathrm{l}$ PBS and analyzed by flow cytometry.

${ }^{51} \mathrm{Cr}$ release assay for $\mathrm{NK}$ cell killing activity. HL-60 cells $\left(2 \times 10^{6} / \mathrm{ml}\right)$ were treated with ATRA or VPA or their combination for $48 \mathrm{~h}$, collected and re-suspended with 100-200 $\mu \mathrm{l}$ complete medium. An amount of $200 \mu 1 \mathrm{Ci}^{51} \mathrm{Cr} / 10^{6}$ target cells was added and incubation was carried out at $37^{\circ} \mathrm{C}$ in $5 \% \mathrm{CO}_{2}$ for $1 \mathrm{~h}$ and mixed once every $10-15 \mathrm{~min}$. Then, the target cells were washed three times with complete medium and adjusted to $1 \times 10^{5}$ cells $/ \mathrm{ml}$ for the subsequent experiment. Then, NK-92 cells were collected and adjusted to $1 \times 10^{7}$ cells/ $\mathrm{ml}$ with complete medium. Target and effector cells $(100 \mu \mathrm{l})(\mathrm{v} /$ $\mathrm{v}=1: 1)$ were seeded on 96-well plates at different cell number ratios (E:T=5:1, 10:1 and 20:1) as the experimental groups. Complete medium $(100 \mu \mathrm{l})$ or $2 \%$ Triton X-100 was added with $100 \mu \mathrm{l}$ target cells to two different wells as spontaneous and maximum release groups, respectively. The final volume was $200 \mu \mathrm{l}$ in each well and three parallel wells were established for each group. Each plate was incubated at $37^{\circ} \mathrm{C}$ in $5 \%$ $\mathrm{CO}_{2}$ for $4 \mathrm{~h}$ and then centrifuged with 1,500 rpm for $10 \mathrm{~min}$. Supernatant (100 $\mu \mathrm{l} /$ well) was added to RIA tubes, and cpm value was detected with a $\gamma$-counter and killing activity was calculated as follows: Cytotoxic activity $(\%)=(\mathrm{cpm}$ values of the experimental group - cpm value of the spontaneous release group)/(cpm value of the maximum release group - cpm value of the spontaneous release group) $\mathrm{x} 100 \%$.

Statistical analysis. Data presented are the means \pm SD from at least three independent experiments and the significant difference between two groups was compared by one-way analysis of variance (ANOVA) followed by Tukey's test using SPSS 13.0 (SPSS, Inc., Chicago, IL, USA). P<0.05 was considered to indicate a statistically significant difference.

\section{Results}

ATRA or VPA inhibits HL-60 cell proliferation. To determine the effect of VPA or ATRA on proliferation, HL-60 cells were treated with various concentrations of the two types of drugs alone, and then cells were stained with trypan blue for different times. The results showed that the number of living cells was gradually decreased with increasing time $(\mathrm{P}<0.05)$ (Fig. 1A), and the number of dead cells was upregulated after ATRA administration. Moreover, the number of living cells was also significantly decreased $(\mathrm{P}<0.05)$ after treatment with VPA at $72 \mathrm{~h}$ (Fig. 1B), with the upregulation of dead cells in the $2 \mathrm{mM}$ VPA group at the indicated time points. Showing time- and dose-dependence, the results revealed that both ATRA and VPA could inhibit the proliferation of HL-60 cells.

ATRA or VPA influence cell cycle distribution of the HL-60 cells. To investigate the influence of ATRA or VPA on cell cycle distribution in the HL-60 cells, various concentrations of these two drugs alone were used to treat HL-60 cells and cell cycle distribution was detected by FACS with PI-staining at different indicated times. The results showed that the percentages of cells in the G0/G1 and S phases within $72 \mathrm{~h}$ after treatment with different concentrations of ATRA had no significant difference when compared with these percentages in the control $(\mathrm{P}>0.05)$. However, the percentage of cells in the $\mathrm{G} 0 / \mathrm{G} 1$ phase was significantly increased after treatment with VPA in a time-dependent manner, and the percentages of cells in the $S$ and $G 2 / M$ phases were exactly reverse $(\mathrm{P}<0.05)$ (Fig. 2). As a result, VPA was be more effective to arrest HL-60 cells in the G0/G1 phase when compared with ATRA.

ATRA or VPA induce the differentiation of HL-60 cells. After the treatment with various concentrations of ATRA, the expression of CD11b on HL-60 cells was increased within $72 \mathrm{~h}$ compared with the control; the effect was particularly significantly at $48 \mathrm{~h}(\mathrm{P}<0.05)$. The results of VPA treatment were similar to that of ATRA administration, and the expression of CD11b was significantly increased in a time- and dose-dependent manner $(\mathrm{P}<0.05)$ (Fig. 3). The results indicated that both ATRA and VPA could effectively induce differentiation of HL-60 cells. 
A
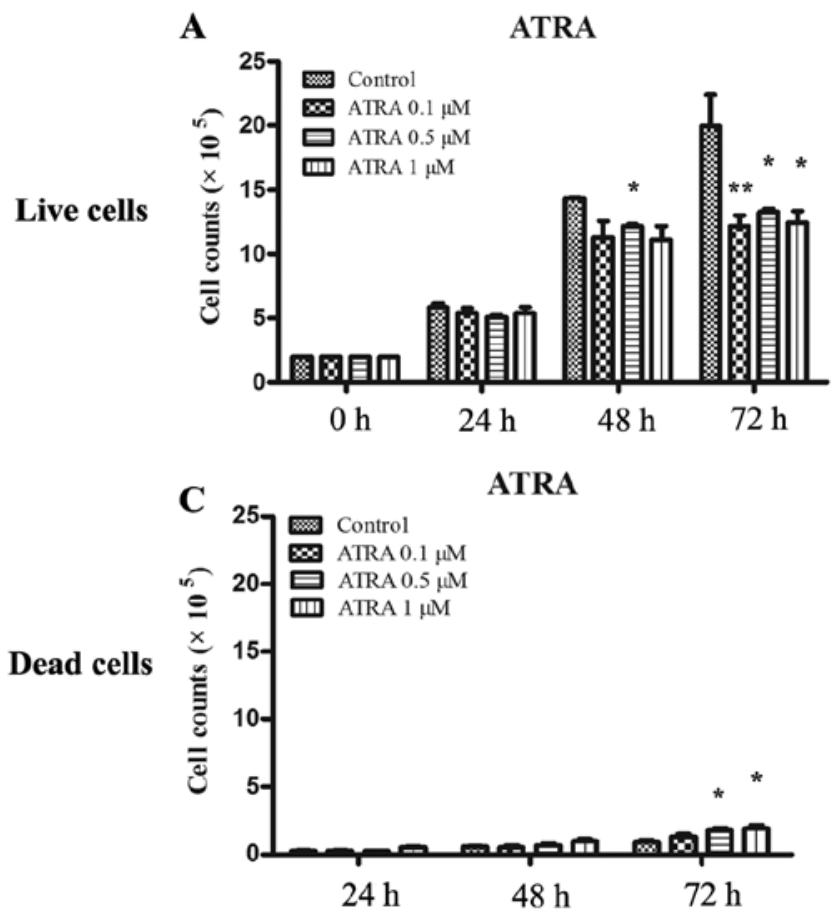

B

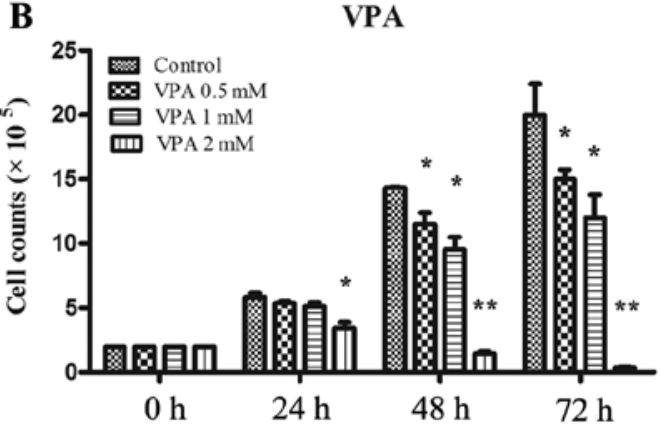

D

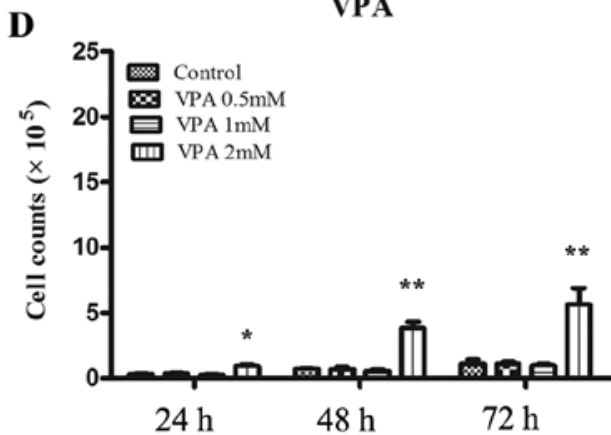

Figure 1. Effect of ATRA and VPA alone on the proliferation of HL-60 cells. HL-60 cells were treated with various concentrations of ATRA or VPA and stained with trypan blue at different times. Both live and dead cells were counted. (A) Live HL-60 cells induced by ATRA. (B) Live HL-60 cells induced by VPA. (C) Dead HL-60 cells induced by ATRA. (D) Dead HL-60 cells induced by VPA. "P<0.05, ${ }^{* *}$ P $<0.01$. ATRA, all-trans-retinoic acid; VPA, valproate.

$$
\text { A }
$$$$
\text { A }
$$

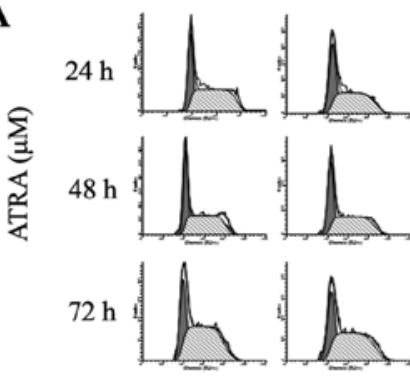

Con

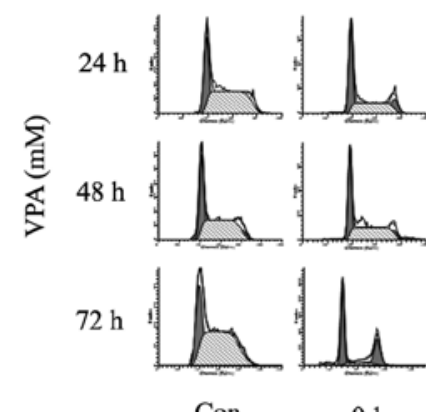

0.1
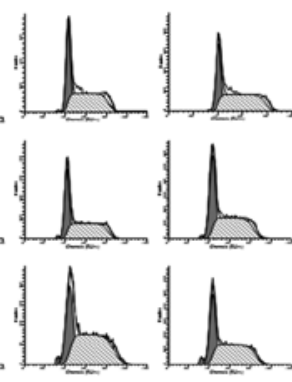

$0.5 \quad 1$
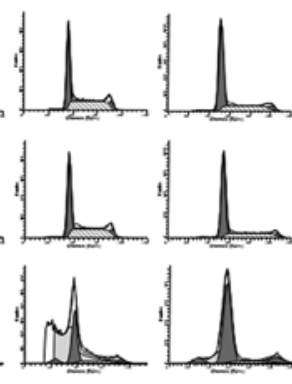

0.5
B

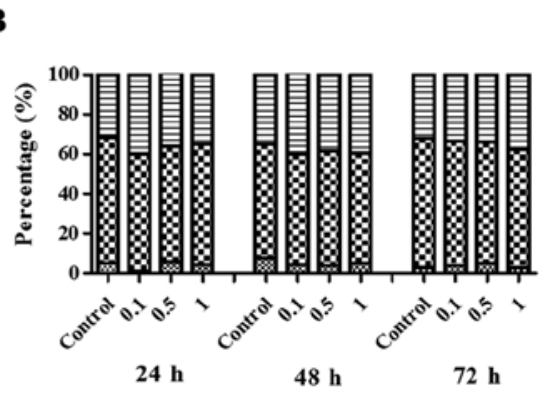

ATRA concentration $(\mu \mathrm{M})$

C

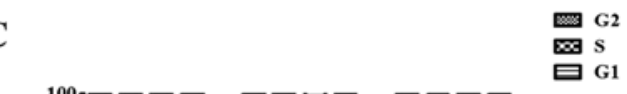

G2

S
G1

Figure 2. Influence of ATRA and VPA alone on HL-60 cell cycle distribution. HL-60 cells treated with ATRA or VPA at the indicated periods were stained with PI, and then the DNA contents and cell cycle distribution were analyzed by FACS. (A) Cell cycle analysis by FACS. The percentage of cell cycle arrest in the G1, S and G2 phases of the HL-60 cells induced by (B) ATRA or (C) VPA. ATRA, all-trans-retinoic acid; VPA, valproate.

Combination of ATRA and VPA synergistically induce HL-60 differentiation. In order to detect the cooperative effect of ATRA and VPA, HL-60 cells were treated with various concentrations of ATRA and VPA alone or in combination for
$48 \mathrm{~h}$. The results showed that the expression of CD11b was increased both in the ATRA and VPA alone groups and the combined groups $(\mathrm{P}<0.05)$ Specifically, the increase noted in the combined groups was more significantly than that of the 
$\mathbf{A}$
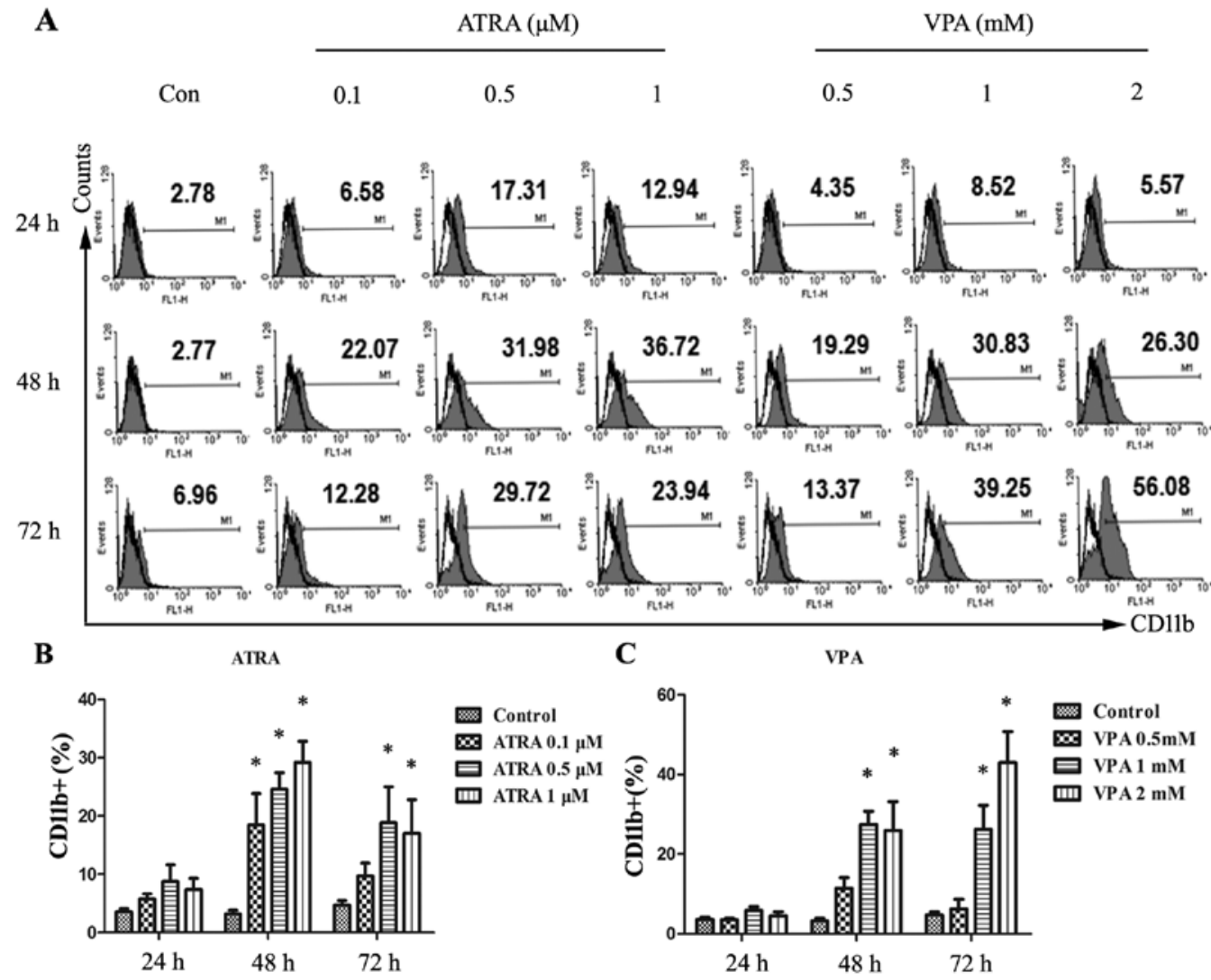

23.94
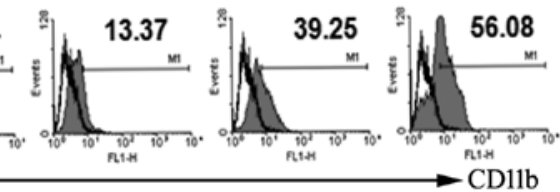

C

VPA

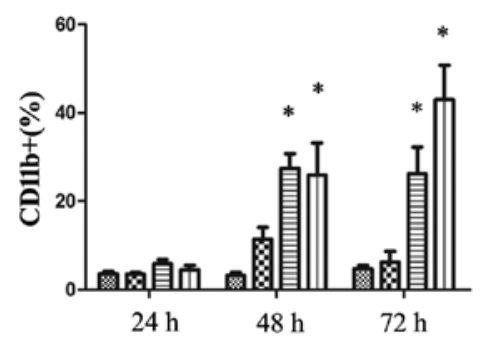

Figure 3. ATRA and VPA alone induce the differentiation of HL-60 cells. HL-60 cells were treated with ATRA or VPA and then were stained with monoclonal anti-CD11b antibodies and analyzed by FACS for the detection of the differentiation marker CD11b. (A) HL- 60 cells $\left(2 \times 10^{5}\right.$ cells/ml) were treated with ATRA $(0.1-0.5 \mu \mathrm{M})$ and VPA $(0.5-1 \mathrm{mM})$ alone or in combination for $48 \mathrm{~h}$, and then were stained with monoclonal anti-CD11b antibodies and analyzed by FACS Average CD11b expression on the HL-60 cells following treatment with (B) ATRA or (C) VPA. Error bars represent SD of three independent experiments. ${ }^{*} \mathrm{P}<0.05$. ATRA, all-trans-retinoic acid; VPA, valproate.

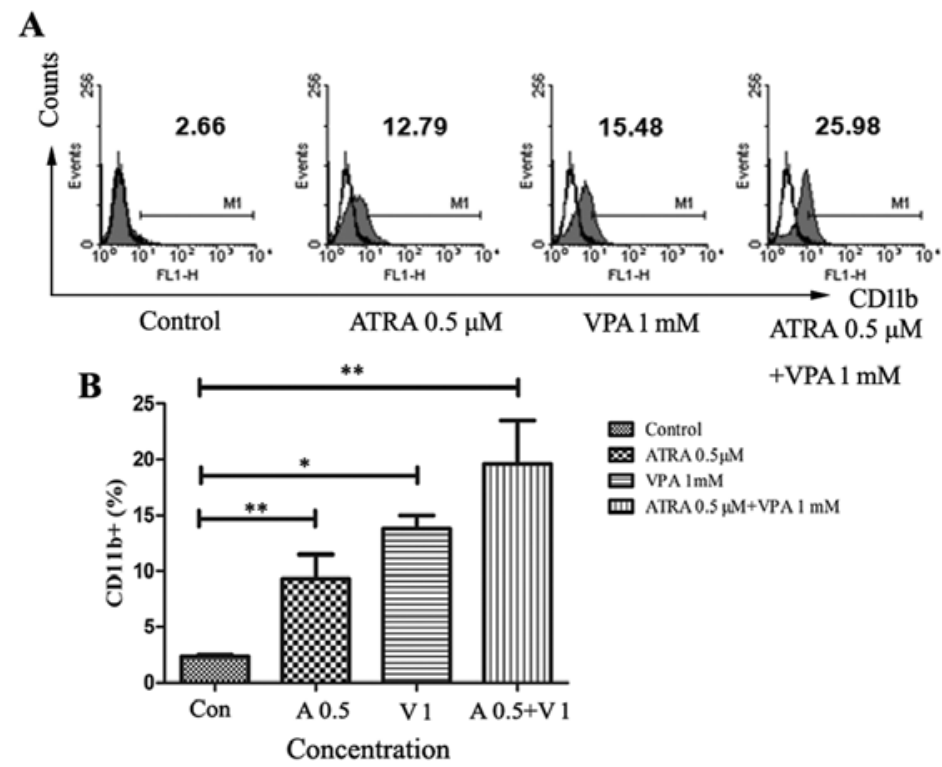

Figure 4. Effect of the treatment with ATRA or VPA alone or with ATRA plus VPA on the induction of the differentiation of HL-60 cells. (A) HL-60 cells $\left(2 \times 10^{5}\right.$ cells $\left./ \mathrm{ml}\right)$ were treated with ATRA $(0.1-0.5 \mu \mathrm{M})$ and VPA $(0.5-1 \mathrm{mM})$ alone or in combination for $48 \mathrm{~h}$, and then were stained with monoclonal antiCD11b antibodies and analyzed by FACS. (B) Average cytotoxicity. Error bars represent SD of three independent experiments. ${ }^{*} \mathrm{P}<0.05,{ }^{* *} \mathrm{P}<0.01$. ATRA, all-trans-retinoic acid; VPA, valproate.

ATRA and VPA alone groups and control groups $(\mathrm{P}<0.05)$, particularly for the VPA $1 \mathrm{mM}$ plus ATRA $0.1 \mu \mathrm{M}$ and VPA
$1 \mathrm{mM}$ plus ATRA $0.5 \mu \mathrm{M}$ combination groups (Fig. 4). This suggested that the two drugs alone and had a synergic effect on 


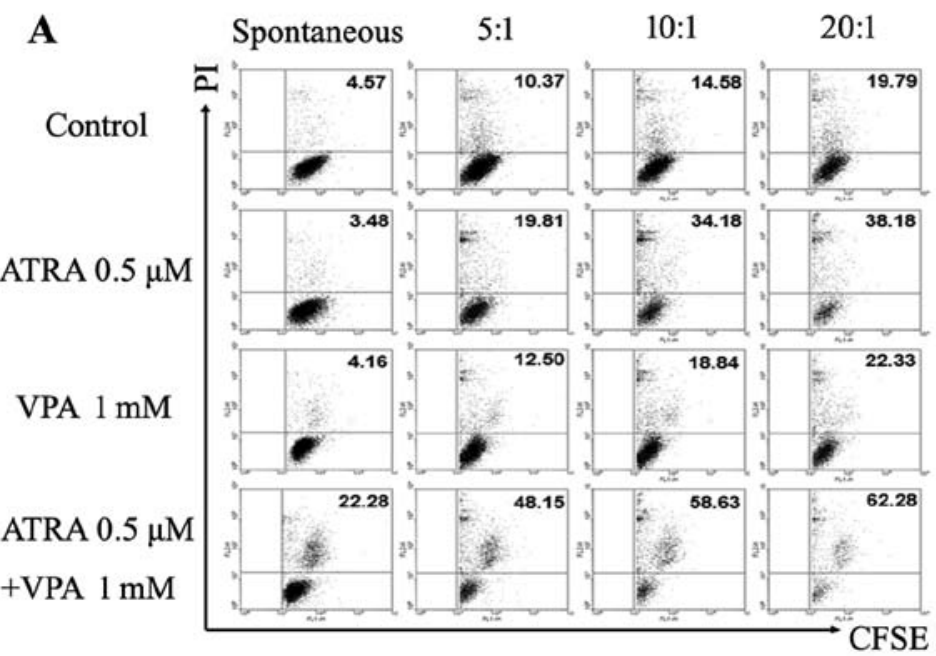

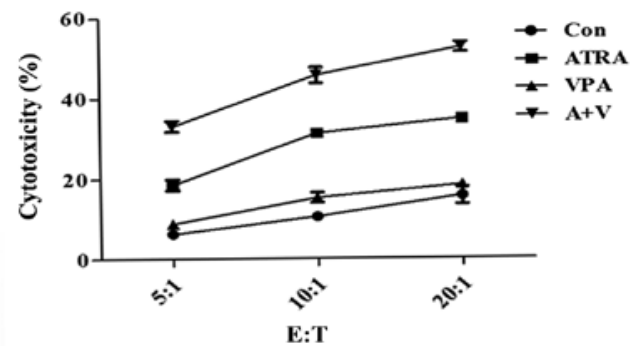

Figure 5. ATRA and VPA enhance the sensitivity of the killing ability of NK cells on the HL-60 cells. Cytotoxicity of NK cells against HL-60 cells was measured at different ratios of NK-92 to HL-60 cells following the various treatments. (A) HL-60 cells were stimulated with $0.5 \mu \mathrm{M}$ ATRA or $1 \mathrm{mM}$ VPA for $48 \mathrm{~h}$, then washed and used as target cells. NK-92 and HL-60 cells (E:T) were used in different ratios. CFSE and PI double staining assay was carried out to assess the killing effect of the NK-92 cells on HL-60 cells. (B) Average cytotoxicity. Error bars represent SD of three independent experiments. ATRA, all-trans-retinoic acid; VPA, valproate.

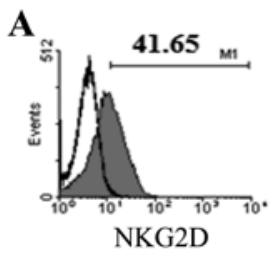

Control

B

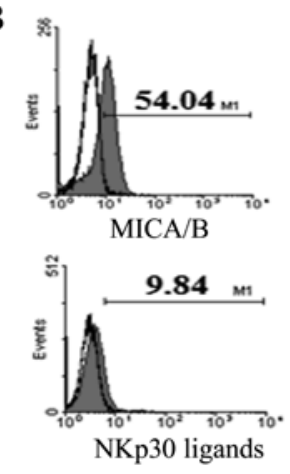

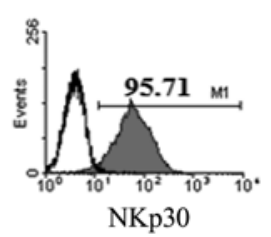

ATRA $0.5 \mu \mathrm{M}$

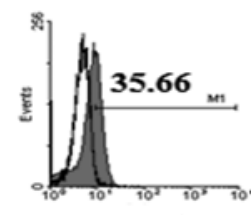

$\mathrm{MICA} / \mathrm{B}$

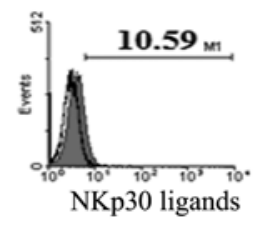

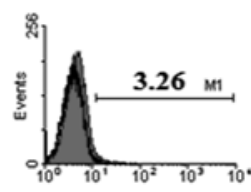

NKG2C

VPA $1 \mathrm{mM}$

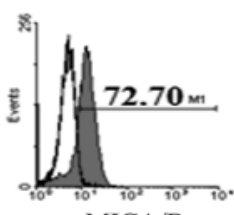

$\mathrm{MICA} / \mathrm{B}$

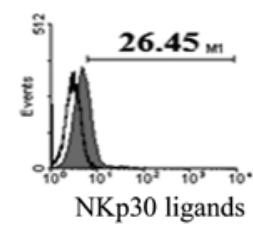

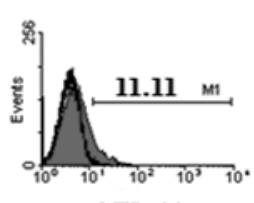

NKp44

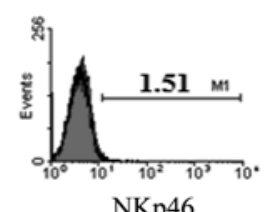

ATRA $0.5 \mu \mathrm{M}+\mathrm{VPA} 1 \mathrm{mM}$

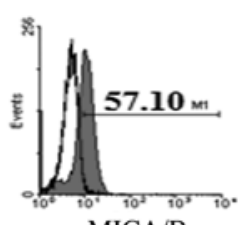

$\mathrm{MICA} / \mathrm{B}$

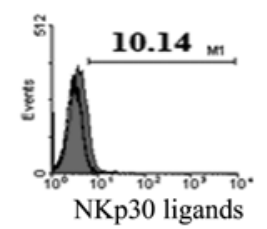

Figure 6. Effect of ATRA and VPA on the expression of activating receptors of NK cells and ligands on HL-60 cells. HL-60 cells were stimulated with $0.5 \mu \mathrm{M}$ ATRA and (or) $1 \mathrm{mM}$ VPA for $48 \mathrm{~h}$. (A) FACS analysis of the expression of the activating receptors on NK-92 cells. (B) The expression of NKG2D and NKp30 ligands on HL-60 cells was measured using FACS. ATRA, all-trans-retinoic acid; VPA, valproate.

the induction of HL-60 differentiation. Therefore, in the next study, we chose the VPA $1 \mathrm{mM}$ plus ATRA $0.5 \mu \mathrm{M}$ group as the model for detecting the sensitivity of HL-60 cells to NK cells.

Enhanced NK cell killing effect of HL-60 cells by ATRA plus $V P A$. To investigate the mechanisms underlying the effect of ATRA plus VPA on HL-60 cells, the killing effect of NK-92 cells on HL-60 cells was detected by FACS after treatment with ATRA and VPA alone or their combination. HL-60 cells were labeled by CFSE and dead cells by PI. HL-60 cells that were double-labeled by CFSE and PI were the cells killed by NK-92 cells. Our results showed that the proportion of doublelabeled cells was greater with an increasing ratio of NK-92 to HL-60 cells and was significant higher in the ATRA and VPA alone groups than that of the control. This indicated that there was an obvious effect of NK-92 cells on HL-60 cells with an increasing E:T ratio. Moreover, the percentage of double-labeled cells treated with the two drugs in combination at the ratio $20: 1$ of NK-92 to HL-60 cells was $62.28 \%$, which was $>19.79 \%$ of the control (Fig. 5A). The percentage of cytotoxicity in the VPA $1 \mathrm{mM}$ plus ATRA $0.5 \mu \mathrm{M}$ combined 
A
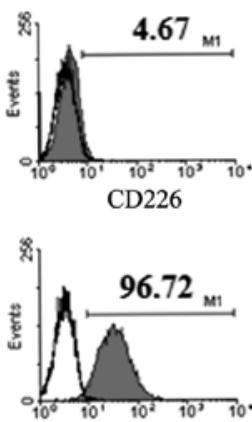

2B4(CD244)
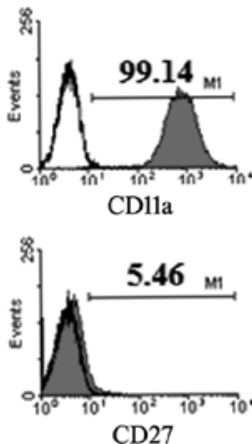

B
ATRA $0.5 \mu \mathrm{M}$
ATRA $0.5 \mu \mathrm{M}$

+ VPA $1 \mathrm{mM}$
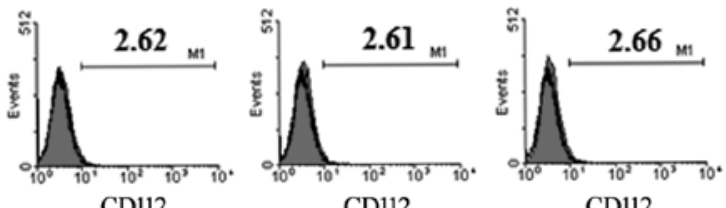

CD112
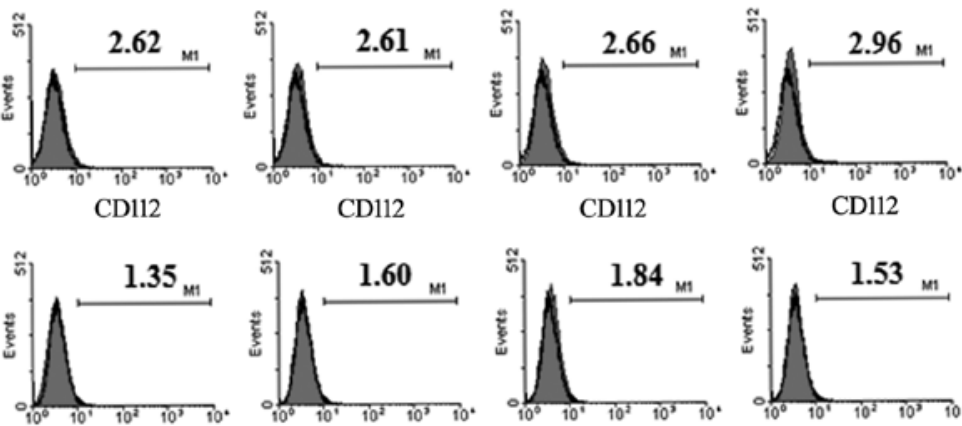

CD48

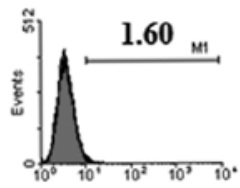

$\mathrm{CD} 48$

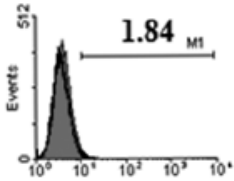

CD48

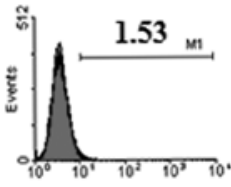

CD48
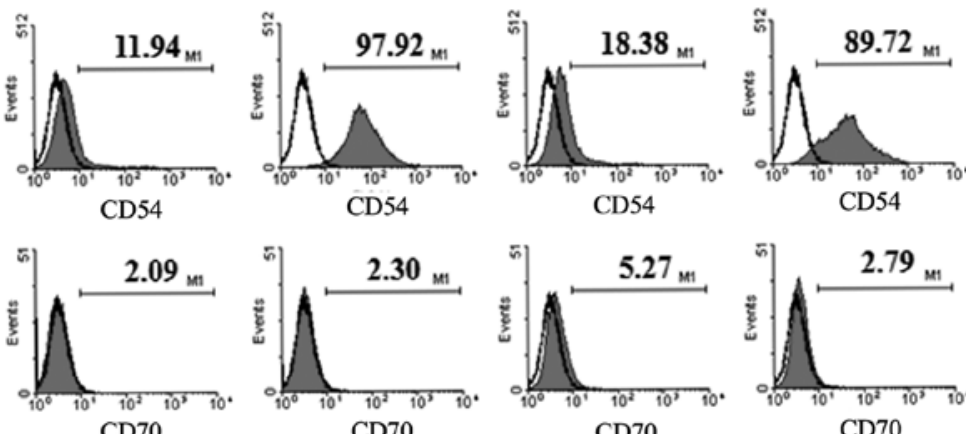

CD70

CD70

Figure 7. Effect of ATRA and VPA on the expression of co-stimulatory and adhesion molecules on HL-60 cells. HL-60 cells were stimulated with $0.5 \mu$ M ATRA and $1 \mathrm{mM}$ VPA alone or in combination for $48 \mathrm{~h}$. (A) FACS analysis of the expression of co-stimulatory and adhesion molecules on NK-92 cells. (B) FACS analysis of the ligands of co-stimulatory and adhesion molecules on HL-60 cells. ATRA, all-trans-retinoic acid; VPA, valproate.

group was the highest compared with ATRA and VPA alone or control groups $(\mathrm{P}<0.01)$ (Fig. 5B). In regards to the enhancment of the sensitivity of leukemia cells to the killing effects of the NK cells, ATRA was more effective than VPA. Following analysis of the effect of the E:T ratio in the ATRA, VPA or their combined group, the range of 5:1 to 20:1 with E:T was found to gradually increase the ability of NK cells to kill HL-60 cells according to each of the three groups. More specifically, the most obvious increase was at the ratio of 20:1 of E:T; the cytotoxicity in the combination group at this ratio achieved $62.28 \%$. From the analysis of the different groups, in the range of 5:1 to 20:1, the cytotoxic activity of NK cells on HL-60 cells in the combination group was significantly higher than that of the single ATRA or VPA group. Furthermore, compared with the control group, ATRA alone, but not the VPA group also had a significant effect on the NK cell activity in the HL-60 cells. Therefore, the combination of ATRA plus VPA should be an ideal way to maintain or promote the susceptibility of HL-60 cells to NK cell-mediated cytolysis.

ATRA and VPA regulate the expression of MHC-I class-related molecules on HL-60 cells. In order to verify whether ATRA and VPA enhance the sensitivity of NK cells by increasing the activation of NK cell activating receptors on NK-92 cells and their ligands on HL-60 cells, we first detected the expression of the activated receptors on NK-92 cells by FACS, including natural cell stimulatory receptors (NCR: NKp46, NKp44 and NKp30), NKG2D and NKG2C. The expression of NKp30 and NKG2D was higher than that of the others (Fig. 6A). However, the expression of ligands corresponding to the receptors on the HL-60 cell surface was different (Fig. 6B). The expression of MICA/B which is one type of ligand of NKG2D on HL-60 cells was significantly increased after VPA treatment alone, but was decreased after ATRA administration only, contrary to each other. Nevertheless, no significant upregulation or downregulation of MICA/MICB was found in the ATRA plus VPA group compared with that of the control. In addition, the expression of ligands of NKp30 on the HL-60 cell surface was not significantly increased (Fig. 6B). The results indicated that MICA/MICB was not chiefly involved in the enhancement of sensitivity of HL-60 cells to NK cells.

ATRA and VPA upregulate the expression of CD54 on HL-60 cells. Next, we analyzed the co-stimulatory and adhesion molecules on NK-92 and their ligands on HL-60 cells. It was found that the expression of CD244 (99.72\%) and CD11a (99.14\%) was higher among CD226, 2B4 (CD244), CD11a and CD27 on the NK-92 cells. Moreover, the expression of CD112, CD48 and CD70 on the HL-60 cells corresponding to CD226, 2B4 (CD244) and CD27 on NK-92 cells had no obvious change after exposure to ATRA and VPA alone or their combination. However, the expression of CD54 on HL-60 cells corresponding to CD11a on NK-92 cells was significantly improved (Fig. 7). These results indicated that the effect of NK cells on HL-60 cells was more likely to be enhanced due to the upregulation of CD54 on HL-60 cells and high level of CD11a on NK cells after exposure to ATRA and/or VPA administration. 
$\mathbf{A}$
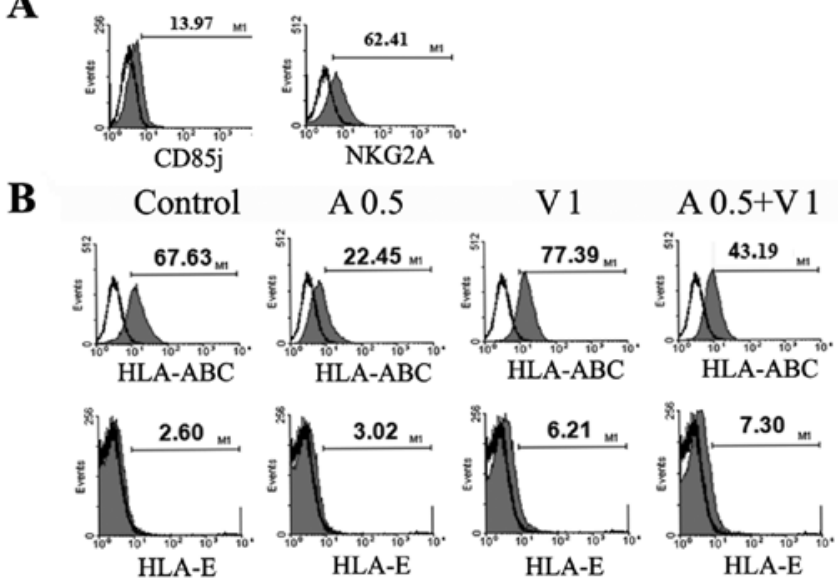

C

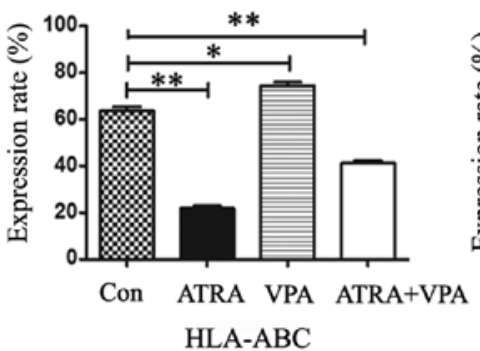

D

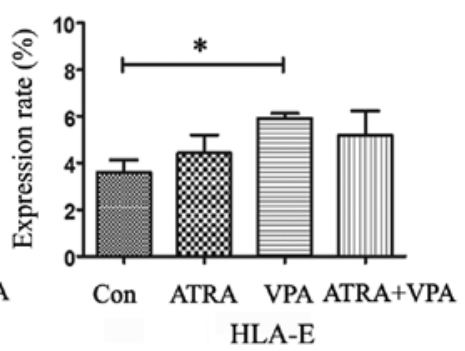

Figure 8. Expression of inhibitory receptor ligands HLA-ABC and HLA-E on HL-60 cells post exposure to ATRA or VPA or their combination. HL-60 cells were stimulated with $0.5 \mu \mathrm{M}$ ATRA or/and $1 \mathrm{mM}$ VPA for $48 \mathrm{~h}$. (A) FACS analysis of the expression of inhibitory receptors on NK-92 cells. (B) Expression of HLA-ABC and HLA-E on the surface of HL-60 cells was measured using FACS. (C) Statistical analysis of HLA-ABC; ${ }^{*} \mathrm{P}<0.05$, ${ }^{* *} \mathrm{P}<0.01$. (D) Statistical analysis of HLA-E; ${ }^{*} \mathrm{P}<0.05$. ATRA, all-trans-retinoic acid; VPA, valproate.

ATRA and VPA decrease the expression of NK cell inhibitory receptor ligands $H L A-A B C$, but slightly increase HLA-E on the HL-60 cell surface. The levels of inhibitory receptor NKG2A and CD85j which are mainly expressed on NK-92 cells were 62.44 and $13.97 \%$, respectively, as determined by FACS analysis. As compared with the control group, the expression of MHC class I molecule HLA-ABC on HL-60 cells was significantly reduced and upregulated by ATRA or VPA, respectively, and still reduced by its combination with ATRA plus VPA. In contrast, as compared with the control group, the expression of HLA-E on HL-60 cells was slightly increased and upregulated after treatment with ATRA or VPA alone, respectively (Fig. 8), while no obvious change was noted in the ATRA plus VPA group. Therefore, HLA-ABC expression was obviously downregulated by exposure to ATRA plus VPA, and the suppression of HLA-E did not significantly change after treatment with ATRA plus VPA.

\section{Discussion}

Acute myeloid leukemia (AML) is characterized by a block of terminal differentiation of the primary hematopoietic stem and progenitor cells at early stages in myelopoiesis (1). However, the classical chemotherapy, ATRA was approved to treat patients with acute promyelocytic leukemia (APL) and most of the patients with APL achieved complete remissions in 1986 (2). Yet, the treatment of ATRA still has some disadvantages such as high recurrence rate and drug resistance $(5,6)$.
Therefore, new novel agents or combination of different drugs were identified to enhance the induction of differentiation and function of immune cells to reduce the relapse rate of APL patients. It was proposed that histone deacetylase inhibitors (HDACIs) are a new type of differentiation-inducing therapy for leukemia (7), VPA as one of the HDACIs alone or in combined with ATRA has been used for the treatment of leukemia, and has achieved good clinical efficacy (8-17). However, it is not clear whether the combination of VPA plus ATRA interfers with the function of immune cells.

VPA, one type of HDACIs, is a short-chain fatty acid used for the clinical treatment of epilepsy with small toxic side-effects (22-24) and plays a role in the treatment of AML alone or in combined with ATRA or other antileukemia agents (12). Previous studies have reported that VPA induced apoptosis and differentiation of leukemia cells and the underlying mechanisms are associated with various oncogenes, tumor-suppressor genes or specific fusion genes in AML $(25,26)$. In the present study, various parameters of HL-60 cells, including proliferation, cell cycle, apoptosis and differentiation, were analyzed in order to re-examine the effect of VPA and/or ATRA on HL-60 cells. The results were consistent with previous studies $(27,28)$ and indicated that ATRA or VPA could significantly inhibit the proliferation of HL-60 cells in a time- and dose-dependent manner. To determine whether the effect of ATRA or VPA on HL-60 cells was associated with cell cycle arrest, we performed cell cycle analysis by flow cytometry. As a result, VPA alone induced a 


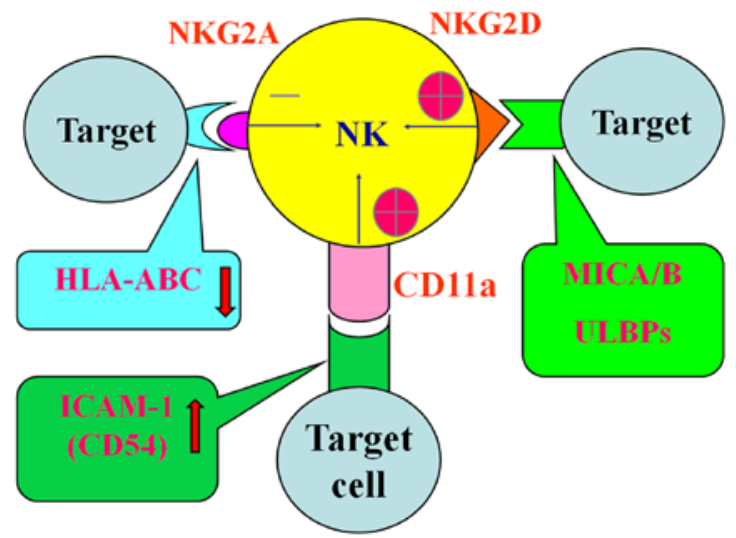

Figure 9. Model image of the enhancement of the susceptibility of HL-60 cells to NK cell-mediated cytolysis induced by ATRA plus VPA. A putative model illustrating the role of CD54 and HL-ABC in the enhancement of the susceptibility of HL-60 cells to NK cell-mediated cytolysis induced by ATRA plus VPA. The combination of ATRA plus VPA obviously increased the sensitivity of HL-60 cells to the killing effects of NK cells, which was chiefly due to the upregulation of CD54 and downregulation of HL-ABC, but was not attributed to NKG2D ligands MICA/MICB whose levels were not modulated significantly. In order to maintain a high level of NK cell-mediated cytolysis in leukemia cells, ATRA plus VPA is an optimal combination treatment. ATRA, all-trans-retinoic acid; VPA, valproate; NK, natural killer.

significant cell accumulation in the G0/G1 phase of the cell cycle with a parallel decrease in the $S$ and $G 2 / M$ phases after treatment for $24 \mathrm{~h}$, and the result was similar to studies by Vrba et al (29). To further investigate whether the effect of these agents on cell growth arrest is associated with differentiation, FACS assay was performed. The results showed that the treatment of ATRA and VPA alone induced the differentiation of HL-60 cells. The combination of ATRA and VPA further augmented the differentiation activity as compared to that of each agent alone. Similar to our results, previous studies demonstrated that VPA induced differentiation in not only HL-60 cells, but also in NB4 and U937 cells, although there were some differences in the induction of differentiation among these leukemia cells $(16,26)$. Kosugi et al also demonstrated that trichostatin A, another HDACi, synergistically induced the differentiation in NB4 and HL-60 cells as well as their ATRA-resistant sublines in combination with ATRA (7). Furthermore, it has been demonstrated that VPA induced differentiation in PML-RAR $\alpha$ and promyelocytic leukemia zinc finger protein (PLZF)-RAR $\alpha$-transformed mouse hematopoietic progenitor cells, and enhanced ATRA-induced differentiation in these cells (15). Thus, these findings suggest that differentiation-inducing activities of these reagents do not appear to be only associated with a specific cytogenetic subtype of AML, and a larger scale study must be launched in order to draw a solid conclusion (16).

In previous studies, it was demonstrated that ATRA or VPA alone could induce differentiation of leukemia cells, and each of these agents also enhanced the susceptibility of leukemia cells to NK cells by upregulating NKG2D ligands MICA/MICB. However, the role of their combination in modulating the function of NK cells and the underlying mechanism had not been elucidated. Generally, NK cells play an important role in antitumor and anti-viral infection, and NKG2D which crosslinks with its ligands (NKG2DL) is the unique activating receptor of NK cells, which may trigger cytotoxic activity of NK cells. VPA significantly enhanced the NK sensitivity of HepG2 (18), HeLa, HepG2 (19) or leukemia cells, and the enhancement by VPA was related to the upregulation of MHC I molecule-associated proteins (MICA and MICB) $(20,21)$.

In the present study, ATRA and VPA alone inhibited the proliferation of HL-60 cells. They could induced a slight G0/G1 phase arrest and significant differentiation induction. We also demonstrated that treatment of HL-60 cells with the combination of $0.5 \mu \mathrm{M}$ ATRA and $1 \mathrm{mM} \mathrm{VPA}$ for $48 \mathrm{~h}$ enhanced the sensitivity of the HL-60 cells to the killing effect of the NK cells (Fig. 5). The notion supported by several previous studies showed that combined ATRA and VPA-induced differentiation of APL cells may be mediated by NK cells $(21,30)$. The expression of NK activating and inhibitory receptor ligands on the HL-60 cell surface was analyzed for their underlying mechanisms. The combination of these two agents increased sensitivity of HL-60 cells to the killing effects of NK cells by downregulating expression of HLA-ABC and upregulating expression of CD54, but not MICA/B (Fig. 9), which was not found in previous studies $(20,21)$. These results provide experimental and clinical basis for the treatment of patients with APL with a combination of low-dose ATRA and VPA, which could induce obvious differentiation and maintain a higher level of function of NK cells.

\section{Acknowledgements}

The present study was supported by the National Natural Science Foundation of China (nos. 81101605, 81172792 and 81573467), the 'Twelfth Five-Year' National Science and Technology Support Program (2013BAI07B02), the Natural Science Foundation of Shandong Province of China (ZR2015YL028 and 2015ZRC03102), the Project for Laureate of Taishan Scholar (no. ts201511075) and the Innovation Project of Shandong Academy of Medical Sciences.

\section{References}

1. Gilliland DG and Tallman MS: Focus on acute leukemias. Cancer Cell 1: 417-420, 2002.

2. Huang ME, Ye YC, Chen SR, Zhao JC, Gu LJ, Cai JR, Zhao L, Xie JX, Shen ZX and Wang ZY: All-trans retinoic acid with or without low dose cytosine arabinoside in acute promyelocytic leukemia. Report of 6 cases. Chin Med J 100: 949-953, 1987.

3. Chen GQ, Shi XG, Tang W, Xiong SM, Zhu J, Cai X, Han ZG, Ni JH, Shi GY, Jia PM, et al: Use of arsenic trioxide $\left(\mathrm{As}_{2} \mathrm{O}_{3}\right)$ in the treatment of acute promyelocytic leukemia (APL): I. $\mathrm{As}_{2} \mathrm{O}_{3}$ exerts dose-dependent dual effects on APL cells. Blood 89: 3345-3353, 1997.

4. Shen ZX, Chen GQ, Ni JH, Li XS, Xiong SM, Qiu QY, Zhu J, Tang W, Sun GL, Yang KQ, et al: Use of arsenic trioxide $\left(\mathrm{As}_{2} \mathrm{O}_{3}\right)$ in the treatment of acute promyelocytic leukemia (APL): II. Clinical efficacy and pharmacokinetics in relapsed patients. Blood 89: 3354-3360, 1997.

5. Dombret H, Castaigne S, Fenaux P, Chomienne C and Degos L: Induction treatment of acute promyelocytic leukemia using all-trans retinoic acid. Controversies about dosage, advantages and side-effect management. Leukemia 8 (Suppl 3): S73-S75, 1994.

6. Tomita A, Kiyoi H and Naoe T: Mechanisms of action and resistance to all-trans retinoic acid (ATRA) and arsenic trioxide $\left(\mathrm{As}_{2} \mathrm{O}_{3}\right)$ in acute promyelocytic leukemia. Int $\mathrm{J}$ Hematol 97: 717-725, 2013. 
7. Kosugi H, Towatari M, Hatano S, Kitamura K, Kiyoi H, Kinoshita T, Tanimoto M, Murate T, Kawashima K, Saito H, et al: Histone deacetylase inhibitors are the potent inducer/enhancer of differentiation in acute myeloid leukemia: A new approach to anti-leukemia therapy. Leukemia 13: 1316-1324, 1999

8. Kuendgen A, Strupp C, Aivado M, Bernhardt A, Hildebrandt B, Haas R, Germing U and Gattermann N: Treatment of myelodysplastic syndromes with valproic acid alone or in combination with all-trans retinoic acid. Blood 104: 1266-1269, 2004

9. Raffoux E, Chaibi P, Dombret H and Degos L: Valproic acid and all-trans retinoic acid for the treatment of elderly patients with acute myeloid leukemia. Haematologica 90: 986-988, 2005.

10. Kuendgen A, Knipp S, Fox F, Strupp C, Hildebrandt B, Steidl C, Germing U, Haas R and Gattermann N: Results of a phase 2 study of valproic acid alone or in combination with all-trans retinoic acid in 75 patients with myelodysplastic syndrome and relapsed or refractory acute myeloid leukemia. Ann Hematol 84 (Suppl 1): S61-S66, 2005.

11. Kuendgen A, Schmid M, Schlenk R, Knipp S, Hildebrandt B, Steidl C, Germing U, Haas R, Dohner H and Gattermann N: The histone deacetylase (HDAC) inhibitor valproic acid as monotherapy or in combination with all-trans retinoic acid in patients with acute myeloid leukemia. Cancer 106: 112-119, 2006.

12. Fredly H, Gjertsen BT and Bruserud O: Histone deacetylase inhibition in the treatment of acute myeloid leukemia: The effects of valproic acid on leukemic cells, and the clinical and experimental evidence for combining valproic acid with other antileukemic agents. Clin Epigenetics 5: 12, 2013.

13. Tassara M, Döhner K, Brossart P, Held G, Götze K, Horst HA, Ringhoffer M, Köhne CH, Kremers S, Raghavachar A, et al Valproic acid in combination with all-trans retinoic acid and intensive therapy for acute myeloid leukemia in older patients. Blood 123: 4027-4036, 2014

14. Zapotocky M, Mejstrikova E, Smetana K, Stary J, Trka J and Starkova J: Valproic acid triggers differentiation and apoptosis in AML1/ETO-positive leukemic cells specifically. Cancer Lett 319: 144-153, 2012.

15. Leiva M, Moretti S, Soilihi H, Pallavicini I, Peres L, Mercurio C, Dal Zuffo R, Minucci S and de Thé H: Valproic acid induces differentiation and transient tumor regression, but spares leukemia-initiating activity in mouse models of APL. Leukemia 26: 1630-1637, 2012.

16. Iriyama N, Yuan B, Yoshino Y, Hatta Y, Horikoshi A, Aizawa $\mathrm{S}$, Takei $\mathrm{M}$, Takeuchi J, Takagi $\mathrm{N}$ and Toyoda $\mathrm{H}$ Enhancement of differentiation induction and upregulation of CCAAT/enhancer-binding proteins and PU.1 in NB4 cells treated with combination of ATRA and valproic acid. Int J Oncol 44: 865-873, 2014

17. Heo SK, Noh EK, Yoon DJ, Jo JC, Park JH and Kim H: Dasatinib accelerates valproic acid-induced acute myeloid leukemia cell death by regulation of differentiation capacity. PLoS One 9: e98859, 2014.

18. Armeanu S, Bitzer M, Lauer UM, Venturelli S, Pathil A, Krusch M, Kaiser S, Jobst J, Smirnow I, Wagner A, et al: Natural killer cell-mediated lysis of hepatoma cells via specific induction of NKG2D ligands by the histone deacetylase inhibitor sodium valproate. Cancer Res 65: 6321-6329, 2005.
19. Zhang C, Wang Y, Zhou Z, Zhang J and Tian Z: Sodium butyrate upregulates expression of NKG2D ligand MICA/B in HeLa and HepG2 cell lines and increases their susceptibility to NK lysis. Cancer Immunol Immunother 58: 1275-1285, 2009.

20. Lu X, Ohata K, Kondo Y, Espinoza JL, Qi Z and Nakao S: Hydroxyurea upregulates NKG2D ligand expression in myeloid leukemia cells synergistically with valproic acid and potentially enhances susceptibility of leukemic cells to natural killer cell-mediated cytolysis. Cancer Sci 101: 609-615, 2010.

21. Poggi A, Catellani S, Garuti A, Pierri I, Gobbi M and Zocchi MR: Effective in vivo induction of NKG2D ligands in acute myeloid leukaemias by all-trans-retinoic acid or sodium valproate. Leukemia 23: 641-648, 2009.

22. Phiel CJ, Zhang F, Huang EY, Guenther MG, Lazar MA and Klein PS: Histone deacetylase is a direct target of valproic acid, a potent anticonvulsant, mood stabilizer, and teratogen. J Biol Chem 276: 36734-36741, 2001.

23. Johannessen CU and Johannessen SI: Valproate: Past, present, and future. CNS Drug Rev 9: 199-216, 2003.

24. Göttlicher M, Minucci S, Zhu P, Krämer OH, Schimpf A, Giavara S, Sleeman JP, Lo Coco F, Nervi C, Pelicci PG, et al: Valproic acid defines a novel class of HDAC inhibitors inducing differentiation of transformed cells. EMBO J 20: 6969-6978, 2001.

25. Liu S, Klisovic RB, Vukosavljevic T, Yu J, Paschka P, Huynh L, Pang J, Neviani P, Liu Z, Blum W, et al: Targeting AML1/ETO-histone deacetylase repressor complex: A novel mechanism for valproic acid-mediated gene expression and cellular differentiation in AML1/ETO-positive acute myeloid leukemia cells. J Pharmacol Exp Ther 321: 953-960, 2007.

26. Cheng YC, Lin H, Huang MJ, Chow JM, Lin S and Liu HE: Downregulation of c-Myc is critical for valproic acid-induced growth arrest and myeloid differentiation of acute myeloid leukemia. Leuk Res 31: 1403-1411, 2007.

27. Deubzer H, Busche B, Rönndahl G, Eikel D, Michaelis M, Cinatl J, Schulze S, Nau H and Witt O: Novel valproic acid derivatives with potent differentiation-inducing activity in myeloid leukemia cells. Leuk Res 30: 1167-1175, 2006.

28. Bartolini G, Orlandi M, Papi A, Ammar K, Tonelli R, Franzoni M, Pession A, Rocchi P and Ferreri AM: Growth inhibition and proapoptotic activity induction by IIF and valproic acid on RA-resistant leukemia cells. Anticancer Res 28: 283-288, 2008.

29. Vrba J, Dolezel P and Ulrichova J: Neutrophilic differentiation modulates the apoptotic response of HL-60 cells to sodium butyrate and sodium valproate. Neoplasma 57: 438-448, 2010.

30. Diermayr S, Himmelreich H, Durovic B, Mathys-Schneeberger A, Siegler U, Langenkamp U, Hofsteenge J, Gratwohl A, Tichelli A, Paluszewska M, et al: NKG2D ligand expression in AML increases in response to HDAC inhibitor valproic acid and contributes to allorecognition by NK-cell lines with single KIR-HLA class I specificities. Blood 111: 1428-1436, 2008. 